\title{
Desenvolvimento comparativo entre sistemas de enchimento na formação e incorporação de defeitos em fundidos de alumínio
}

\section{Comparative development between systems of formation and incorporation of defects in aluminum castings}

\footnotetext{
${ }^{1}$ Centro Universitário SOCIESC - UNISOCIESC, Departamento Instituto Superior Tupy, Joinville, Santa Catarina, Brasil.

${ }^{2}$ Fundição Tupy AS, Departamento Engenharia Metalúrgica, Joinville, Santa Catarina, Brasil.

${ }^{3}$ Instituto Anima, Departamento Laboratório de Fundição, Joinville, Santa Catarina, Brasil.

e-mail: preti@unisociesc.com.br, orlpreti@gmail.com, alexandre.ribeiro@tupy.com.br, ibereroberto@gmail.com
}

\section{RESUMO}

O presente estudo compara os sistemas de enchimento de moldes de fundição convencional e tipo faca (contrito), avaliando o desempenho na formação e incorporação de defeitos (bolhas de ar) na fabricação de peça fundida em alumínio. Foram fabricados modelos na forma de placas (10 mm x $150 \mathrm{~mm}$ x $200 \mathrm{~mm}$ ) e com os sistemas de enchimento emplacados, para a elaboração dos moldes pelo processo cura a frio (fenol uretânica). Foi fundida a liga AA 356.0, em forno tipo poço a cadinho com capacidade de $60 \mathrm{~kg}$. A temperatura de vazamento foi de $710 \pm 10^{\circ} \mathrm{C}$. Para caracterização, empregou-se análise em software de simulação numérica (MAGMASOFT), ensaio de visual e de Raio-X. Os resultados mostraram que as amostras do sistema convencional apresentaram maior tendência à formação e incorporação de defeitos, ficando evidente o melhor desempenho do sistema de enchimento tipo faca.

Palavras-chave: Alumínio; Enchimento tipo Convencional e Faca; Defeitos.

\section{ABSTRACT}

The present work is a comparative study between two filling systems, one called conventional and another called constrict. The objective of this work is to evaluate the performance of the systems in the formation and incorporation of defects (oxides and air bublles) in aluminum cast part (plate), in no bake process. In this study, we used numerical simulation software (MAGMASOFT), X-ray test, tensile test, optical microscopy and scanning electron microscopy (SEM). In comparison, the samples of conventional system showed greater tendency to the formation and incorporation of defects and lower mechanical resistance, showing better performance of constrict system.

Keywords: Aluminium; Conventional Filling and Knife; Defect.

\section{INTRODUÇÃO}

Dentre os metais mais fundidos no Brasil, o alumínio e suas ligas encontram-se em $3^{\circ}$ lugar, atrás apenas dos ferros e aços fundidos, mostrando a importância deste metal frente à demanda produtiva existente [1], contendo baixa densidade, boa resistência à corrosão, elevada condutibilidade térmica e elétrica e boa combinação de propriedades mecânicas.

O processo de fundição de peças em alumínio e suas ligasé realizado em moldes dispensáveis e/ou permanentes, empregando-se areia e ligas ferrosas, respectivamente. São constituídos por sistema de enchimento, em que a forma e dimensões dos canais (descida, distribuição e ataque) são essenciais para levar o metal líquido até a cavidade onde que se formará a peça e promovem também o controle do fluxo do metal liquido (lamelar ou turbulento), que influencia na qualidade da peça. 
A turbulência do metal líquido no sistema de canais pode promover defeitos como bolhas de ar e inclusões não metálicas (óxidos, areia, entre outros) [2]. Dependendo do projeto e dimensionamento dos canais os defeitos podem surgir em menor ou maior quantidade, prejudicando a sanidade interna, qualidade das propriedades mecânicas e acabamento superficial.

Segundo Kolososki [3], os defeitos de fundição em ligas de alumínio fundido, devem-se às características do metal líquido e do projeto do sistema de enchimento e alimentação, sendo os mais comuns os defeitos de contração tipo rechupe [4], as microporosidades de contração ou de hidrogênio, bolhas de ar e inclusões de óxidos.

As microporosidades, de acordo com Fuoco [5], são formadas por dois mecanismos: a) alimentação inadequada, resultando em poças de líquido interdendrítico; b) a segregação de hidrogênio para as últimas porções de alumínio líquido, conforme mostra a Figura 1 [6].

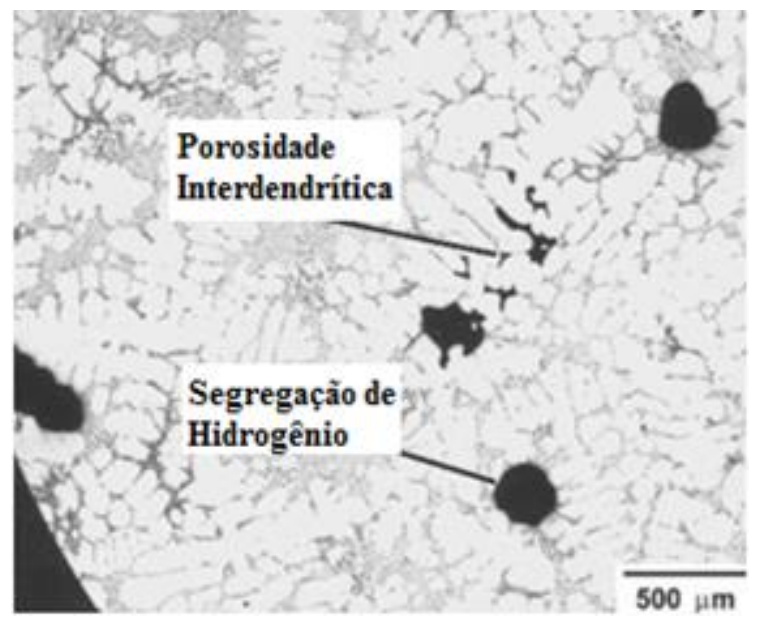

Figura 1: Microporosidade em liga de alumínio silício [6].

A incorporação de defeitos de bolhas de ar ocorre principalmente pela turbulência do líquido no sistema de canais. Outros defeitos podem ser causados pelo dobramento de uma superfície líquida oxidada [3, 5, 6]. Os óxidos, presentes na forma de filmes, podem ser dobrados, quebrados e incorporados ao banho quando da existência de turbulência [8].

Os sistemas de enchimento podem atenuar ou agravar a formação e incorporação de defeitos. Assim, o projeto e dimensionamento dos canais é considerado um fator determinante para obtenção de peças da qualidade do fundido [4].

No enchimento do molde normalmente implicam em elevadas velocidades do metal devido a altura de vazamento ser superiore à 12,5 mm, o que promove turbulência. De acordo com Campbell [9], a velocidade máxima de um fluxo de alumínio líquido é de $500 \mathrm{~mm} / \mathrm{s}$ (equação 1), para não haver rompimento do filme de óxido e sua incorporação à peça, indicando que fluxo turbulento é algo comum para os sistemas de enchimento de fundição.

$$
V=\alpha(2 \cdot g \cdot h)^{1 / 2}
$$

Onde:

$V=$ velocidade do líquido em queda livre $(\mathrm{mm} / \mathrm{s})$

$g=$ aceleração da gravidade $\left(9,810 \mathrm{~mm} / \mathrm{s}^{2}\right) ;$

$h=$ altura da queda livre ( $\mathrm{mm})$.

$\alpha=$ fator de perdas por atrito (tipicamente empregado 0,5 para fundição de alumínio).

A literatura $[8,10,11]$ recomenda que os sistemas de enchimento não pressurizados para ligas de alumínio devem: a) usar canais de descida cônicos (não retos); b) uso de bacia na base do canal de descida; c) evitar alterações bruscas de seção transversal e de direção do fluxo de metal; d) utilizar relações entre seções 
dos canais típicas (1:2:2, 1:2:4 ou 1:4:4); e) usar os canais de distribuição no molde inferior e ataques no molde superior e f) preenchimento da cavidade que formará a peça dentro do molde por baixo.

A partir da equação 2 [12], determina-se as áreas dos canais que segue a seguinte ordem: a) Área da base do canal de descida; b) Área do canal de distribuição e c) Área do canal de ataque. Esta ralação pode ser convergente (sendo, seção reguladora do fluxo o canal de ataque - Ex.: $1: 0,8: 0,6$ ) ou divergente (cenário se inverte - Ex.: 1:2:2)

$$
A=\frac{P}{\rho . t . \alpha \sqrt{2 g h}}
$$

Onde:

$A$ = área da seção de choque $\left(\mathrm{mm}^{2}\right)$;

$P=$ peso do conjunto, peça mais massalote $(\mathrm{g})$;

$\rho=$ densidade do metal fundido $\left(\mathrm{g} / \mathrm{mm}^{3}\right)$;

$t=$ tempo de enchimento $(\mathrm{s})$.

Fuoco e Correa [7] destacam que a operação de vazamento promove a geração de um tubo de óxido em torno do metal; que a velocidade na base do canal de descida pode atingir $3.000 \mathrm{~mm} / \mathrm{s}$, necessitando diminuí-la nos canais de distribuição e ataque.

Para garantir a sanidade do fundido, o sistema de enchimento deve ser constrito às paredes dos canais quando a velocidade for superior a $500 \mathrm{~mm} / \mathrm{s}$, reduzi-la abaixo deste antes de atingir a cavidade que resulta a peça $[7,9]$.

Rezvani, Yang e Campbell [13] propuseram os canais finos e largos de 3 a 6 mm [3] de espessura (tipo faca), minimizando problemas de canais parcialmente preenchidos, mesmo quando usado sistemas despressurizados. Os canais tipo faca, aumentam as forças de atrito, reduz a turbulência do metal líquido e a aspiração de ar, devido à menor probabilidade de formação de zona de baixa pressão [7].

Mantendo a constrição do metal líquido às paredes dos canais, reduz-se a velocidade do metal (perda de energia cinética) e, consequentemente, inibe a formação de uma superfície turbulenta [14] e, maiores reduções de velocidade do metal líquido podem ser obtidas quando aplicadas sucessivas curvaturas $\left(90^{\circ}\right)$ ao longo do canal $[7,12]$.

\section{MATERIAIS E MÉTODOS}

Para a realização deste estudo, adotou-se a geometria da amostra tipo placa $10 \mathrm{~mm}$ x $150 \mathrm{~mm}$ x $200 \mathrm{~mm}$, sendo que os cálculos das áreas dos canais de descida, distribuição e ataque foram realizados conforme equação 2 e mostrados na Tabela 1 e Figura $2(a, b)$ do sistema convencional e tipo faca, respectivamente.

Tabela 1: Dimensões e características dos sistemas de enchimento convencional e faca.

\begin{tabular}{c|c|c}
\hline Sistema de Enchimento & Convencional & Faca \\
\hline Relação entre áreas & $1: 2: 2$ & $1: 1: 1$ \\
\hline Área canal de distribuição $\left(\mathrm{mm}^{2}\right)$ & $338(13 \times 26 \mathrm{~mm})$ & $338(6,5 \times 52 \mathrm{~mm})$ \\
\hline Canal de distribuição $\left(\mathrm{mm}^{2}\right)$ & $676(26 \times 26 \mathrm{~mm})$ & $342(76 \times 4,5 \mathrm{~mm})$ \\
\hline Canal de distribuição após primeiro ataque $\left(\mathrm{mm}^{2}\right)$ & $338(26 \times 13 \mathrm{~mm})$ & $171(38 \times 4,5 \mathrm{~mm})$ \\
\hline Canal de ataque $\left(\mathrm{mm}^{2}\right)$ & $333676(37 \times 9 \mathrm{~mm})$ & $172(43 \times 4 \mathrm{~mm})$ \\
\hline
\end{tabular}

A Figura (2) mostra os dois conjuntos de sistemas de enchimento, sendo o tipo convencional (Figura 1(a)) e tipo faca (Figura 1(b)), este último mantendo-se a relação 1:1:1 com menor espessura e maior largura nos canais de distribuição e de ataque. 

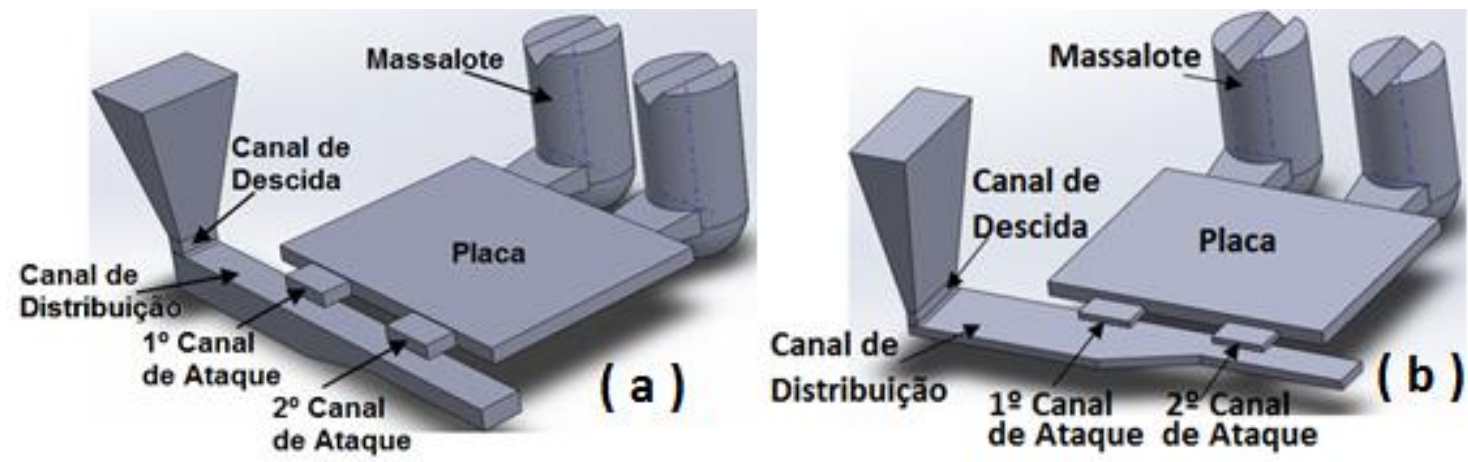

Figura 2: Modelamento do sistema de enchimento convencional (a) e faca (b).

A simulação de enchimento dos sistemas convencional e faca foi realizada com auxílio do Software MAGMA $^{5}$, versão 5.3, sendo avaliados pelos recursos: a) "air entrapment", permite visualizar os pontos com tendência a aprisionamento de ar/bolhas, b) "flow trace", permite entender o fluxo de enchimento em função do tempo; c) "temperature", permite verificar as temperaturas ao longo da peça em função do tempo e, d) "velocity", permite avaliar a velocidade de enchimento do molde com metal líquido $(\mathrm{mm} / \mathrm{s})$ ao longo da peça, em função do tempo.

Os dados de entrada utilizados no software foram: a) temperatura de vazamento de $740{ }^{\circ} \mathrm{C}$; b) liga de alumínio silício AA 356.0; c) granulometria da areia de 60 AFS; d) processo de areia tipo cura a frio/sílica; e) tempo para desmoldagem de 900 segundos; f) tempo de enchimento do molde de 3 segundos; g) altura entre panela e abertura do canal de descida utilizando o vazamento junto ao molde.

Foram produzidos 7 moldes para cada sistema de enchimento em processo de moldagem cura a frio (fenol uretânico), sendo a liga AA 356.0 (Tabela 2) fundida em forno tipo cadinho com capacidade de $50 \mathrm{~kg}$, aquecido a gás natural. A liga no estado líquido passou pelos processos de escorificação com $0,5 \%$ de sal Alfaflux AL 12C - Fabricante: Alfatrend, desgaseificação com nitrogênio aplicado com o equipamento MEGA SPM - Fabricante: Alfatrend, controle de temperatura com pirômetro modelo Forneiro Kal da Italterm e vazamento realizado em temperatura de $740^{\circ} \mathrm{C}$.

As placas foram desmoldadas, separando os sistemas de enchimento e alimentação após tempo de 900 segundos e seguido de limpeza com jato de granalha por 5 minutos.

As placas foram inspecionadas visualmente para avaliar os aspectos superficiais (presença de defeitos e marcas de fluxo de metal) e pelo ensaio não destrutivo de Raio-X, com o auxílio do equipamento de marca Gilardoni que detecta defeitos acima de $0,4 \mathrm{~mm}$.

\section{RESULTADOS}

\subsection{Simulação do enchimento}

A Figura 3, mostra o resultado da simulação que permite verificar as temperaturas ao longo do sistema de enchimento convencional (Figura (3a)) e faca (Figura (3b)) da placa fundida em função do tempo durante o enchimento do molde.

É observado na Figura (3a) que o sistema de enchimento convencional, mantem o metal em temperaturas mais elevadas durante todo o enchimento, ou seja, apresenta baixa perda térmica (há menor tendência de falha por metal frio) em relação ao sistema de enchimento tipo faca (Figura (3b)), que apresenta menor temperatura. 


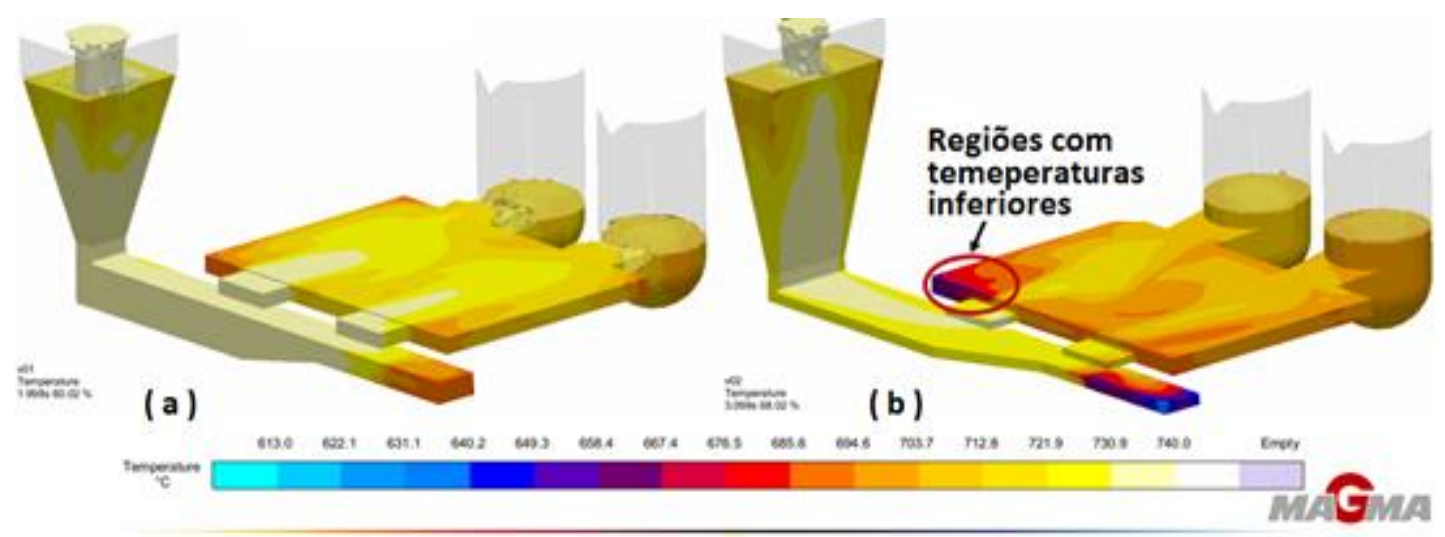

Figura 3: Temperatura de enchimento do molde com sistema de enchimento convencional (a) e faca (b).

Na Figura (3b), na região circulada, apresenta a maior perda térmica, mas não foi indicado defeitos do tipo solda fria.

A Figura (4) e a Tabela 2, mostram os resultados da simulação que permite verificar as velocidades do metal líquido (m/s) em quatro regiões ao longo do sistema de enchimento convencional (Figura (4a)) e faca (Figura (4b)) da placa fundida durante o enchimento do molde.

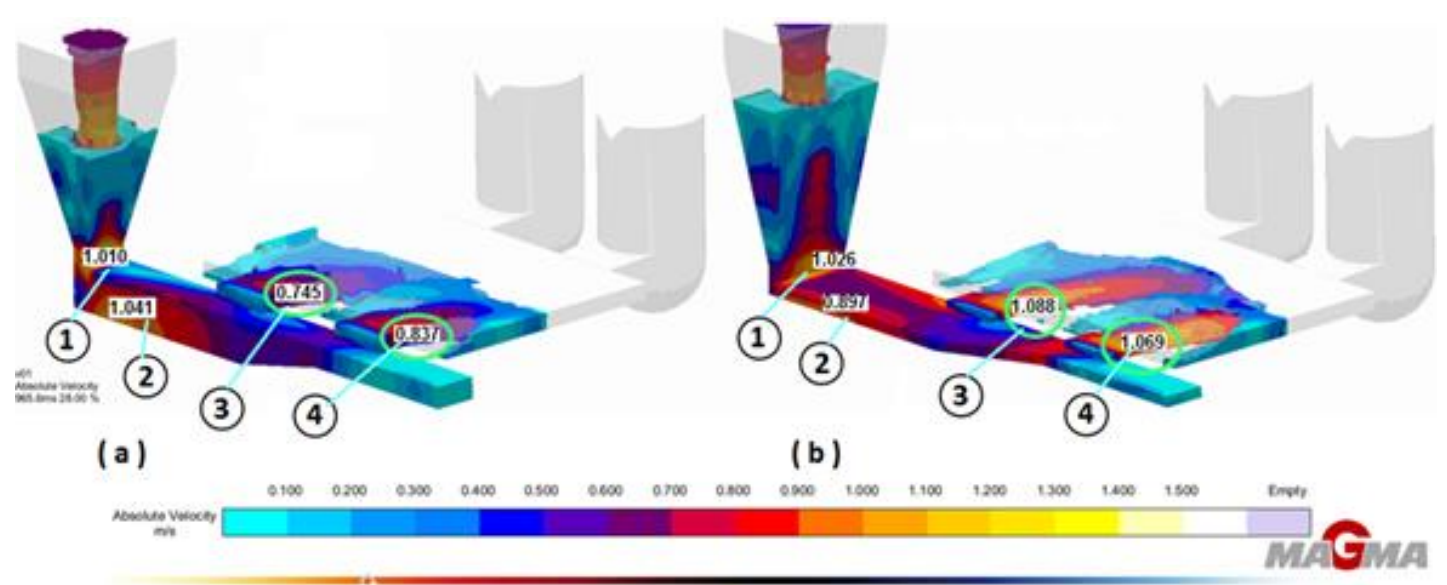

Figura 4: Velocidades do metal líquido durante o enchimento dos moldes.

A Tabela 2 mostra os valores das velocidades do metal liquido durante o processo de enchimento (no caso feito por simulação) do molde para o sistema convencional e o tipo faca.

Tabela 2: Velocidade nos canais dos sistemas de enchimento convencional e tipo faca

\begin{tabular}{|c|c|c|c|}
\hline Região & Descrição & Sistema de enchimento & Velocidade $(\mathrm{m} / \mathrm{s})$ \\
\hline \multirow{2}{*}{1} & \multirow{2}{*}{ Seção de choque } & Convencional & 1,010 \\
\hline & & Faca & 1,026 \\
\hline \multirow{2}{*}{2} & \multirow{2}{*}{ Canal de distribuição } & Convencional & 1,041 \\
\hline & & Faca & 0,897 \\
\hline \multirow{2}{*}{3} & \multirow{2}{*}{ Canal de ataque 01} & Convencional & 0,745 \\
\hline & & Faca & 1,088 \\
\hline \multirow{2}{*}{4} & \multirow{2}{*}{ Canal de ataque 02} & Convencional & 0,837 \\
\hline & & Faca & 1,069 \\
\hline
\end{tabular}


Com os resultados mostrados na Tabela 2, pode-se considerar que as velocidades na seção de choque (Região 1) foram similares para ambos os sistemas de enchimento. A velocidade do metal no canal de distribuição (Região 2) é menor para o sistema faca, provavelmente devido a mudança abrupta de direção e associada ao maior atrito com as paredes dos canais, estando de acordo com Fuoco e Correa [7].

A velocidade no canal de ataque (Região 3 e 4) no sistema faca é maior quando comparada ao sistema convencional, este fato pode ser justificado pelo canal tipo faca apresentar redução expressiva na espessura do canal de ataque (estrangulamento).

A Figura (5) mostra os resultados da simulação que permite verificar os fluxos do metal líquido ao longo do sistema de enchimento convencional (Figura (5a)) e faca (Figura (5b)) da placa fundida durante o enchimento do molde.

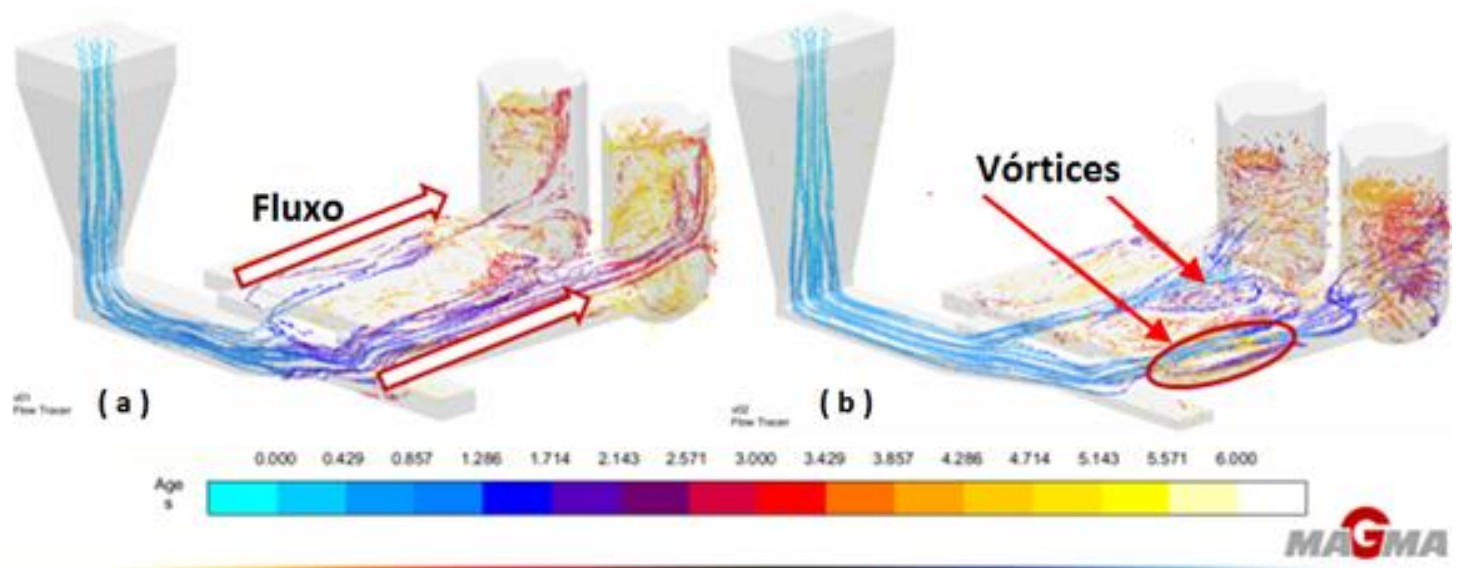

Figura 5: Fluxo do metal líquido durante o enchimento dos moldes.

A Figura (5), mostra que o fluxo do metal líquido no sistema de enchimento convencional apresentou maior direcionamento aos massalotes, com menor formação de vórtices em relação ao sistema de enchimento tipo faca. A formação dos vórtices pode ser atribuída principalmente à maior velocidade do metal líquido no canal de ataque e, pode promover a formação e incorporação de inclusões e óxidos.

A Figura (6) mostra os resultados da simulação que permite verificar os pontos com tendência a aprisionamento de ar/bolhas ao longo do sistema de enchimento convencional (Figura (6a)) e faca (Figura (6b)) da placa fundida durante o enchimento do molde.

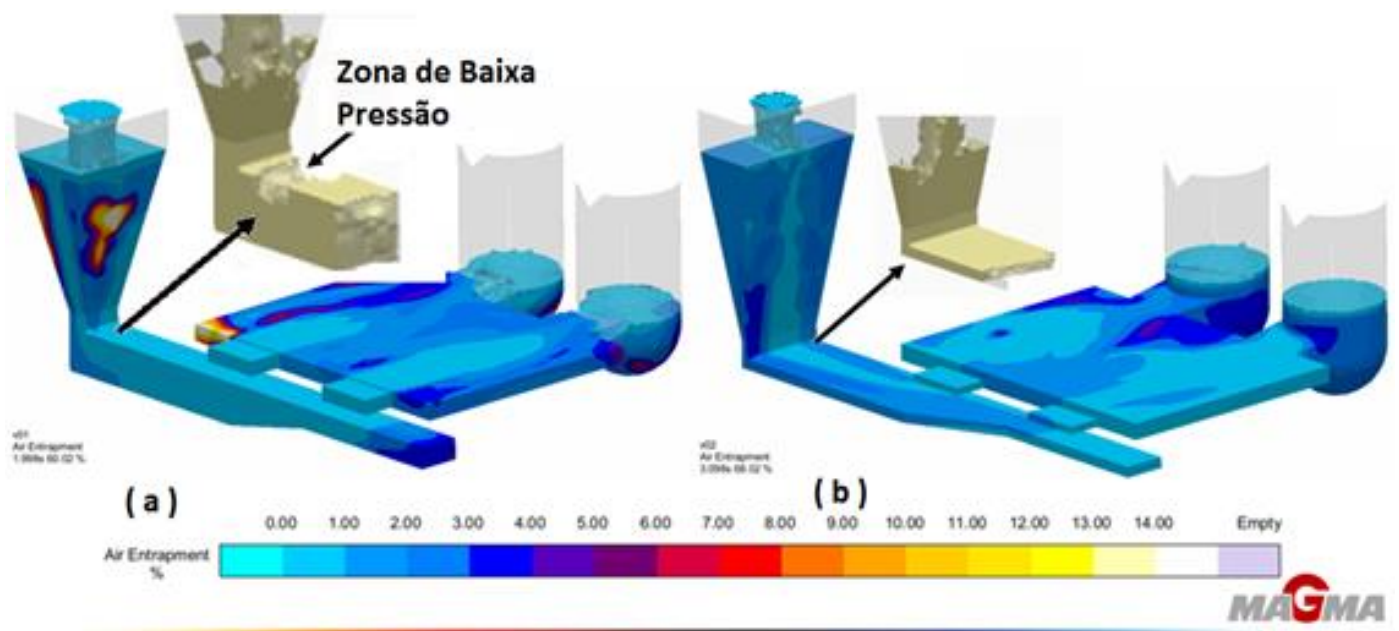

Figura 6: Análise de aprisionamento de ar nos sistemas de enchimento. 
A Figura (6) mostra que o sistema tipo faca tem menor tendência ao aprisionamento de ar em relação ao sistema de enchimento convencional. Este comportamento pode ser justificado pela ausência da zona de baixa pressão no sistema de enchimento faca, colaborando também para menor tendência à oxidação e incorporação de óxidos.

A Figura (7) mostra detalhes da simulação da solidificação do conjunto peça em que se observa que o rechupe se concentrou nos dois massalote.

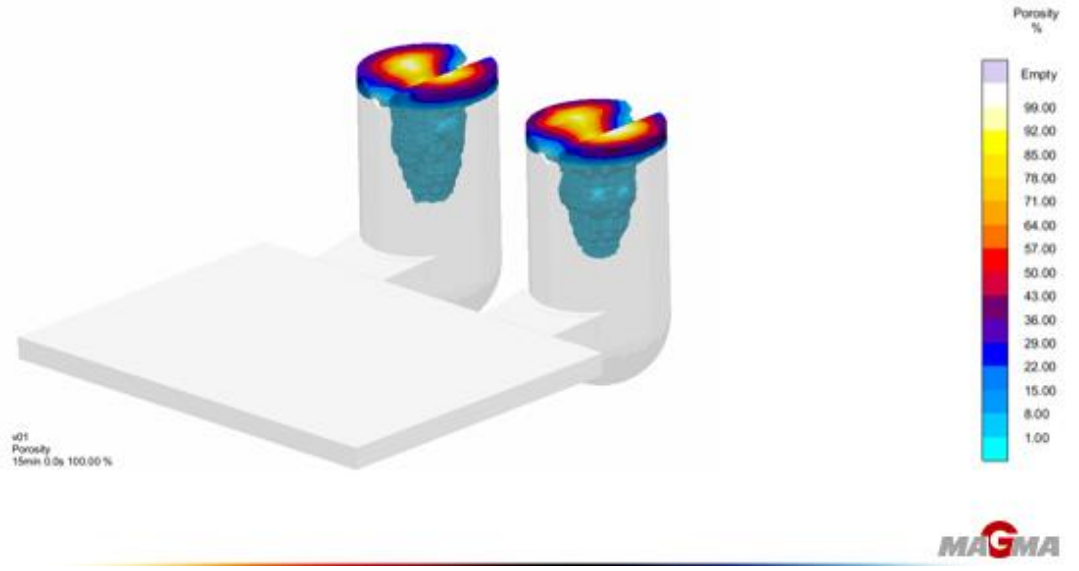

Figura 7: Avaliação de solidificação do conjunto peça e massalotes.

Observando os dados da Figura 7, permiti verificar que o dimensionamento dos massalotes é eficaz para a não formação de rechupes na placa fundida, tanto para o sistema de enchimento convencional (Figura (7a)) e em faca (Figura (7b)).

\subsection{Análise química}

A tabela 3 mostra a composição química da liga AA 356.0 e da obtida a partir de amostras extraídas de uma das placas fundidas.

Tabela 3: Composição da liga AA 356.0 e obtida

\begin{tabular}{c|c|c|c|c|c|c|c|c}
\hline & $\mathrm{Si}$ & $\mathrm{Fe}$ & $\mathrm{cu}$ & $\mathrm{Mn}$ & $\mathrm{Mg}$ & $\mathrm{Zn}$ & $\mathrm{Ti}$ & $\mathrm{Al}$ \\
\hline AA 356.0 & 6,50 & $0,60^{*}$ & $0,25^{*}$ & $0,35^{*}$ & $0,20-0,45$ & $0,35^{*}$ & $0,25^{*}$ & $\mathrm{Bal}$ \\
\hline Obtida & 6,51 & 0,37 & 0,24 & 0,07 & 0,45 & 0,03 & 0,03 & $\mathrm{Bal}$ \\
\hline *Valores máximos; \% em massa
\end{tabular}

A tabela 2 mostra que a composição química da liga fundida e, que foi produzida as amostras, está dentro da faixa de valores indicados pela norma AA 356.0.

\subsection{Ensaio não Destrutivo (RX)}

A Figura (8) mostra uma placa do sistema de enchimento convencional que apresentou três defeitos abertos à superfície na parte superior da placa, visto na inspeção visual. As demais placas não apresentaram defeitos superficiais visíveis. Também todas as placas apresentaram enchimento completo e nenhum outro defeito típico de fundição (solda fria, escama, veiamento, entre outros). 


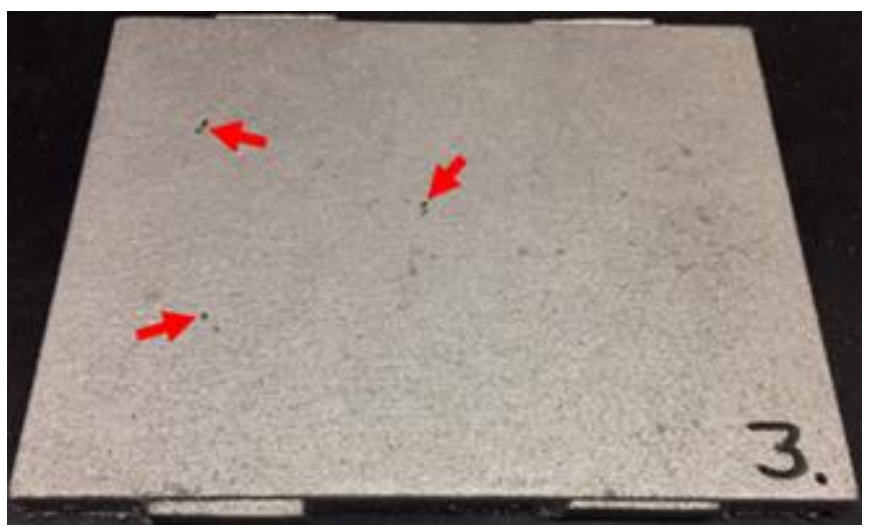

Figura 8: Defeitos superficiais na parte superior da placa obtida no sistema convencional.

$\mathrm{Na}$ inspeção por Raio-X, foi detectado defeitos internos em todas as placas produzidas pelo sistema de enchimento convencional (Figura (9)) e, em maior número do que no sistema de enchimento tipo faca, conforme mostra a Tabela 4.

Tabela 4: Quantidade de defeitos nas placas.

\begin{tabular}{c|c|c|c|c|c|c|c|c|c|c|c|c|c|c}
\hline Sistema de enchimento & \multicolumn{1}{c|}{ Convencional } & \multicolumn{1}{c}{ Faca } \\
\hline Placa & 1 & 2 & 3 & 4 & 5 & 6 & 7 & 8 & 9 & 10 & 11 & 12 & 13 & 14 \\
\hline No defeitos $^{\text {o }}$ & 3 & 11 & 17 & 3 & 6 & 5 & 3 & 0 & 4 & 0 & 2 & 1 & 3 & 0 \\
\hline Média & \multicolumn{1}{|c|}{6,9} \\
\hline
\end{tabular}

A Figura (9) mostra apenas as placas que apresentaram maior número de defeitos interno dos sistemas de enchimento convencional (Figura (9a)) e em faca (Figura (9b)), detectado pela inspeção por Raio-X, caracterizados como bolhas de ar, sendo de ordem milimétrica [9].
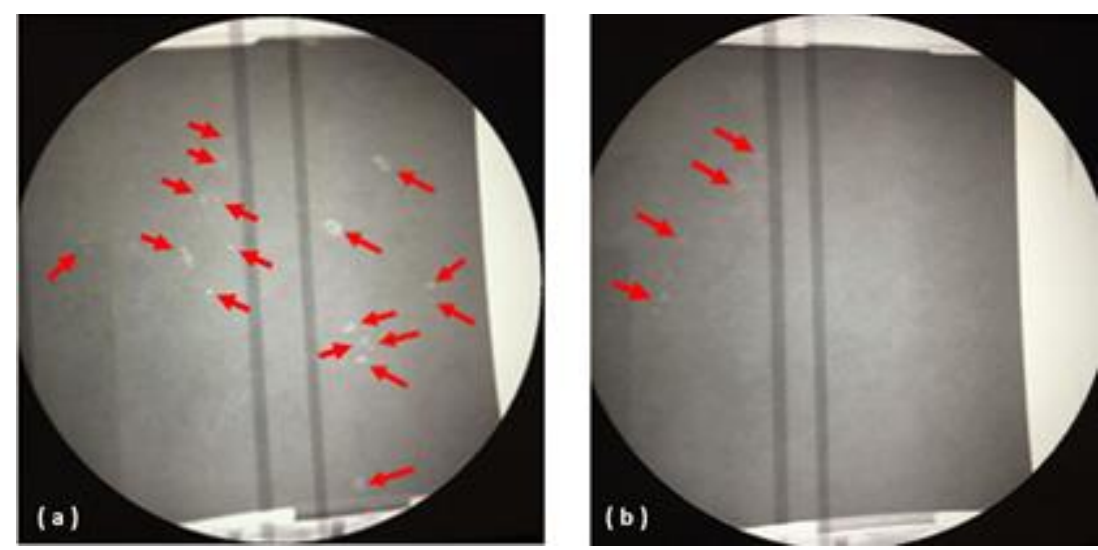

Figura 9: Defeitos internos: a) placa do sistema de enchimento convencional e b) tipo faca.

A maior quantidade de defeitos tipo bolha de ar nas placas obtidas pelo sistema de enchimento convencional, deve-se possivelmente, pela maior incorporação de ar devido a zona de baixa pressão formada na base do canal de decida (detalhe na Figura (6a)). 


\section{CONCLUSÕES}

Em relação aos sistemas de enchimento convencional e faca, utilizados neste estudo, para o enchimento de placas em posição horizontal em liga de alumínio AA 356, utilizando a ferramenta de simulação Magma, pode-se dizer que:

a) O sistema de enchimento convencional proporciona menor perda térmica e menor velocidade de deslocamento do metal líquido nos canais de ataque em relação ao sistema de enchimento tipo faca.

b) No sistema de enchimento convencional o fluxo de metal líquido é mais direcional ao massalote do que o sistema de enchimento tipo faca, promovendo menor efeito vórtices.

c) No sistema de enchimento convencional ocorre a formação de região de baixa pressão na base do canal de decida, que possibilita a entrada de ar, promovendo a incorporação e formação de defeitos tipo bolha de ar.

d) De acordo com a simulação feita com o software Magma, para o sistema de enchimento convencional, evidenciou-se a formação de zona de baixa pressão, gerando maior quantidade (6,9 em média) de defeitos do tipo bolhas de ar em relação ao sistema de enchimento tipo faca (1,4 em média).

Os resultados deste estudo mostram que o sistema de enchimento tipo faca, apresenta melhor desempenho em reduzir a formação e incorporação de defeitos do tipo bolhas de ar.

\section{AGRADECIMENTOS}

Magma Engenharia do Brasil e Aciaria Frederico Missner (Luiz Alves/SC), nas simulações de enchimento com o Software MagmaSoft. Modelação Jurk, na fabricação do modelo de fundição. Fundição UNISOCIESC, confecção dos moldes em areia. Fundição Rosil, fusão da liga e obtenção das placas fundidas. Fundição Wetzel - Alumínio por realizar ensaio de raio-x.

\section{BIBLIOGRAFIA}

[1] ABIFA. Desempenho do setor de Fundição Março/2015.

[Http://abifa.org.br/wpcontent/uploads/2015/04/desempenho_marco.pdf]. São Paulo: ABIFA, 2017. [Acesso em: 02 marços 2017]. Disponível em: http://abifa.org.br

[2] WANG, Q.G, APELIAN, D, LADOS, D.A., "Fatigue behavior of A356/357 aluminum cast alloys, Part II - Effect of microstructural constituents", Journal Of Light Metals, v. 1, pp. 85-97, 2001.

[3] KOLOSOSKI, J., "Estudo de sistema de canais para fundição de ligas de alumínio por gravidade", Dissertação de mestrado, Universidade Estadual de Campinas, Campinas, SP, Brasil, 2001.

[4] DUARTE, I. R., Fundição, Sistema de alimentação, SOCIESC, Joinville, 2013.

[5] FUOCO, R., Curso defeitos de fundição de origem metalúrgica em ligas alumínio-silício, ABAL, 1997.

[6] WANG, Q. G., CREPEAU, P. N., DAVIDSON, C. J., et al., Oxide films, pores and the fatigue lives of cast aluminum alloys, Metallurgical And Materials Transactions, v. 37, pp. 887-895, 2006.

[7] FUOCO, R., CORRÊA, E.R., Estudo de sistema de canais para fundição de ligas de alumínio por gravidade, São Paulo, 2003.

[8] CAMPBELL, J., Castings. 2 ed., Oxford, Butterworth Heinemann, 2003.

[9] CAMPBELL, J., Complete Casting Handbook, Oxford, Butterworth Heinemann, 2011.

[10] AMERICAN FOUNDRYMEN'S SOCIETY, Aluminium Casting Technology, 2 ed., Des Plaines, AFS, 1993.

[11] ASM INTERNATIONAL, Casting, ASM Handbook, v. 15, 9 ed., Ohio, 2004.

[12] MARIOTTO, C. L., ALBERTIN, E., FUOCO, R., Sistemas de enchimento e alimentação de peças fundidas, 1 ed. Associação Brasileira de Metais (ABM), São Paulo, 1987.

[13] REZVANI, M., YANG, X., CAMPBELL, J., The effect of ingate design on the strenght \& reliability of Al castings, 1999.

[14] DAI, X., et al., "Effects of runner system design on the mechanical strength of Al-7Si-Mg alloy castings", Materials Science and Engineering A, 354, pp. 315-325, 2003. 
BIBLIOGRAFIA

Orlando Preti

Alexandre Guilherme Ribeiro

Iberê Roberto Duarte
https://orcid.org/0000-0002-3096-704X

https://orcid.org/0000-0003-2060-7320

https://orcid.org/0000-0002-3949-7945 